\title{
Collisional and viscous damping of MHD waves in partially ionized plasmas of the solar atmosphere
}

\author{
M. L. Khodachenko ${ }^{1}$, T. D. Arber ${ }^{2}$, H. O. Rucker ${ }^{1}$, and A. Hanslmeier ${ }^{3}$ \\ 1 Space Research Institute, Austrian Academy of Sciences, Schmiedlstraße 6, 8042 Graz, Austria \\ e-mail: maxim. khodachenko; helmut.rucker@oeaw.ac.at \\ 2 Physics Department, University of Warwick, Coventry, CV4 7AL, UK \\ e-mail: t.d.arber@warwick.ac.uk \\ 3 Institute for Geophysics, Astrophysics and Meteorology, Universtitätsplatz 5, 8010 Graz, Austria \\ e-mail: arnold.hanslmeier@uni-graz.at
}

Received 18 August 2003 / Accepted 6 April 2004

\begin{abstract}
Magnetohydrodynamic (MHD) waves are widely considered as a possible source of heating for various parts of the outer solar atmosphere. Among the main energy dissipation mechanisms which convert the energy of damped MHD waves into thermal energy are collisional dissipation (resistivity) and viscosity. The presence of neutral atoms in the partially ionized plasmas of the solar photosphere, chromosphere and prominences enhances the efficiency of both these energy dissipation mechanisms.

A comparative study of the efficiency of MHD wave damping in solar plasmas due to collisional and viscous energy dissipation mechanisms is presented here. The damping rates are taken from Braginskii 1965 and applied to the VAL C model of the quiet Sun (Vernazza et al. 1981). These estimations show which of the mechanisms are dominant in which regions. In general the correct description of MHD wave damping requires the consideration of all energy dissipation mechanisms via the inclusion of the appropriate terms in the generalized Ohm's law, the momentum, energy and induction equations. Specific forms of the generalized Ohm's Law and induction equation are presented that are suitable for regions of the solar atmosphere which are partially ionised.
\end{abstract}

Key words. magnetohydrodynamics (MHD) - waves - Sun: atmosphere - Sun: photosphere - Sun: chromosphere

\section{Introduction}

Magnetohydrodynamic (MHD) waves are believed to play an important role in the solar atmosphere. In particular, they are widely considered as a possible source for heating of various parts of the outer solar atmosphere (Piddington 1956; Osterbrock 1961; Gordon \& Hollweg 1983; Hollweg 1986, 1991; Narain \& Ulmschneider 1996; Goodman 2000, 2001). The heating effect of MHD waves depends on dissipation mechanism which convert the energy of damped MHD waves into thermal energy.

It is well known that the presence of neutral atoms in partially ionized plasmas enhances the dissipation of MHD waves. The energy and momentum transfer from the Alfvén waves damped in the partially ionized chromospheric plasma has been proposed as a driving factor for solar spicules (Haerendel 1992; DePontieu \& Haerendel 1998; James \& Erdélyi 2002; James et al. 2003). The leakage of Alfvén wave energy through the footpoints of a coronal loop, caused by wave dissipation in the partially ionized chromosphere (DePontieu et al. 2001; Ofman 2002), is considered as one of possible explanations of the recently observed rapid damping of coronal loop oscillations (Aschwanden et al. 1999b; Nakariakov et al. 1999).

Analysis of the distribution of temperature and heat deposition along magnetic loops based on recent SOHO/EIT and TRACE EUV observations shows that, contrary to the soft X-ray loops observed by Yohkoh/SXT (Priest et al. 1998), cooler loops are heated non-uniformly (Aschwanden et al. 1999a, 2000, 2001). More heat deposition is detected near the loop footpoints. Dissipation of slow magnetoacoustic waves in the chromospheric parts of the loop was suggested as a possible explanation of such non-uniform energy deposition (Nakariakov et al. 2000; Tsiklauri \& Nakariakov 2001). The non-uniform heating of the chromospheric footpoints of a magnetic loop also appears as a key assumption of the dynamical model for prominence formation in a dipped magnetic arcade (Antiochos et al. 1999; Karpen et al. 2001). It is also invoked in explanations of material flows and intensity variations in nonflaring coronal loops (Spadaro et al. 2003).

Therefore, understanding the specifics of MHD wave propagation and damping in the partially ionized plasmas of the photosphere and chromosphere is important for understanding 
the heating and dynamics of the whole external solar atmosphere. The same concerns the case of another typical example of the partially ionized solar plasmas, - the material of prominences. MHD waves are widely referred regarding the interpretation of the material motion in the prominences, their possible oscillations and energy budget.

In previous publications the damping of MHD waves has been considered using two different energy dissipation mechanisms. One group of researchers assumes the friction between ions and the neutral fraction in the partially ionized plasma is the dominant dissipation mechanism (Piddington 1956; Haerendel 1992; DePontieu \& Haerendel 1998; DePontieu et al. 2001; James \& Erdélyi 2002). Another group of authors takes into account only the viscosity effects (Gordon \& Hollweg 1983; Nakariakov et al. 2000; Tsiklauri \& Nakariakov 2001; Ofman 2002).

It is worth emphasizing the different physical nature of the above two mechanisms of MHD wave dissipation. The forces associated with the viscosity have purely kinetic origin and are caused by the momentum transfer during the thermal motion of particles. The collisional friction forces appear due to the averaged relative motion of the plasma species. Therefore, the correct description of MHD wave damping requires the consideration of both energy dissipation mechanisms via the inclusion of the appropriate terms in the generalized Ohm's Law, as well the momentum and energy equations.

While the frictional dissipation (electrical resistivity) can usually be safely neglected as compared to viscosity effects in the fully ionized solar corona (Gordon \& Hollweg 1983), the relation and mutual role of both these dissipation mechanisms for the damping of different types of MHD waves in the lower (partially ionized) solar atmosphere requires a special comparative study. In this paper we give some elementary estimations of the frictional and viscous damping of MHD waves and discuss which dissipation mechanism is dominant in different regions of the solar atmosphere.

\section{MHD wave damping in the linear approximation}

In this section we summarize the results from Braginskii (1965) on the damping rates of MHD waves. These results are used in later sections and are reproduced here for reference. The method adopted by Braginskii was to calculate the energy decay time for different modes from the local heating rates $Q_{\text {visc }}, Q_{\text {frict }}$, etc. In this approach each damping mechanism is assumed to be linearly independent and therefore the method is only formally valid for light damping. The decay of wave amplitude is described by the complex frequency $\omega-\mathrm{i} \omega \delta$ so that the energy in a particular wave mode will decay as $\mathrm{e}^{-t / \tau}$, where $\tau=(2 \omega \delta)^{-1}$ is a characteristic wave damping time and $\delta$ is the logarithmic damping decrement. Damping rates in this paper are therefore presented in terms of $2 \omega \delta$ and represent energy decay times. Throughout the paper, unless explicitly stated otherwise, the temperature is in energy units $\left(T[\operatorname{erg}] \equiv k_{\mathrm{B}} T\left[\mathrm{~K}^{\circ}\right]\right)$, the magnetic field in Gauss and all other quantities are in cgs.

The aim of this paper is a comparative study of the collisional friction and viscous damping of MHD waves. We limit ourselves here to a consideration of only these two energy dissipation mechanisms and do not take into account MHD wave damping due to thermoconductivity effects.

The MHD wave modes are the Alfvén wave, fast magnetoacoustic, and slow magnetoacoustic waves. The slow magnetoacoustic wave degenerates in the limit $C_{\mathrm{s}} \ll V_{\mathrm{A}}$ (or the plasma $\beta=\frac{4 \pi p_{0}}{B_{0}^{2}} \ll 1$ ) to a pure acoustic wave distorted by the magnetic field. The frequency of this wave is $\omega=C_{\mathrm{s}} k_{\|}$. The frequency of fast magnetoacoustic waves in this case is $\omega=V_{\mathrm{A}} k$, not to be confused with the Alfvén frequency which is $\omega=V_{\mathrm{A}} k_{\|}$. Here $V_{\mathrm{A}}=\frac{B_{0}}{\sqrt{4 \pi \rho_{0}}}$ and $C_{\mathrm{s}}=\left(\frac{\gamma p_{0}}{\rho_{0}}\right)^{1 / 2}$ are the Alfvén and the sound speed in a plasma with total density $\rho_{0}$, pressure $p_{0}$, external magnetic field $B_{0}$, and adiabatic constant $\gamma$. The condition $C_{\mathrm{s}} \ll V_{\mathrm{A}}$ holds true for magnetic fields $B_{0}>10 \mathrm{G}$ everywhere above the middle chromosphere, as well as in the majority of prominences.

In the opposite limit of $C_{\mathrm{s}} \gg V_{\mathrm{A}}$ (or plasma $\beta \gg 1$ ), which is valid in the non- or weakly magnetized $B_{0}<10 \mathrm{G}$ regions of the chromosphere and photosphere, fast magnetoacoustic waves transform to the usual acoustic wave with frequency $\omega=C_{\mathrm{s}} k$, while the slow magnetoacoustic wave behaves like the Alfvén wave with frequency $\omega=V_{\mathrm{A}} k_{\|}$.

\subsection{The case of fully ionized plasma}

For the damping decrements of Alfvén, fast magnetoacoustic, and acoustic waves propagating in a fully ionized plasma, Braginskii (1965) gives the following expressions:

Alfvén wave (A.w.):

$2 \omega \delta_{\text {Joule }}^{\text {A.w. }} \equiv \frac{1}{\tau_{\text {Joule }}^{\text {A.w. }}}=\frac{c^{2}}{4 \pi \sigma_{\|}} k_{\perp}^{2}+\frac{c^{2}}{4 \pi \sigma_{\perp}} k_{\|}^{2}$,

$2 \omega \delta_{\text {visc }}^{\text {A.w. }} \equiv \frac{1}{\tau_{\text {visc }}^{\text {A.w. }}}=\frac{1}{\rho_{0}}\left(\eta_{1} k_{\perp}^{2}+\eta_{2} k_{\|}^{2}\right)$.

Fast magnetoacoustic (or magnetosonic) wave (f.ms.w.):

$2 \omega \delta_{\text {Joule }}^{\text {f.ms.w. }} \equiv \frac{1}{\tau_{\text {Joule }}^{\text {f.ms.w. }}}=\frac{c^{2}}{4 \pi \sigma_{\perp}} k^{2}$,
$2 \omega \delta_{\text {visc }}^{\text {f.ms.w. }} \equiv \frac{1}{\tau_{\text {visc }}^{\text {f.ms.w. }}}=\frac{1}{\rho_{0}}\left[\left(\frac{\eta_{0}}{3}+\eta_{1}\right) k_{\perp}^{2}+\eta_{2} k_{\|}^{2}\right]$.

Acoustic (or sound) wave (s.w.):

$2 \omega \delta_{\text {Joule }}^{\text {s.w. }} \equiv \frac{1}{\tau_{\text {Joule }}^{\text {s.w. }}}=\frac{c^{2}}{4 \pi \sigma_{\perp}} k_{\perp}^{2} \frac{C_{\mathrm{s}}^{2}}{V_{\mathrm{A}}^{2}}$,

$2 \omega \delta_{\text {visc }}^{\text {s.w. }} \equiv \frac{1}{\tau_{\text {visc }}^{\text {s.w. }}}=\frac{1}{\rho_{0}}\left(\frac{4}{3} \eta_{0} k_{\|}^{2}+\eta_{2} k_{\perp}^{2}\right)$.

Since in a fully ionized plasma the frictional heat term contains only the Joule heating part, i.e. $Q_{\text {frict }}=Q_{\text {Joule }}=\frac{j_{\|}^{2}}{\sigma_{\|}}+\frac{j_{\perp}^{2}}{\sigma_{\perp}}$, we 
use the notation $\delta_{\text {Joule }}$ in place of $\delta_{\text {frict }}$ in the above expressions for damping decrements from Braginskii (1965).

In Equations (1)-(6) $k_{\|}$and $k_{\perp}, \sigma_{\|}$and $\sigma_{\perp}$ are components of the wave vector and electric conductivity of the plasma in directions parallel and perpendicular to the background magnetic field respectively; $\eta_{0}, \eta_{1}$, and $\eta_{2}$ are viscosity coefficients. In the case of an isothermal plasma the viscosity coefficients are determined essentially by ions. Braginskii (1965) gives the following expressions for the ion viscosity coefficients:

$\eta_{0}=0.96 n_{\mathrm{i}} T_{\mathrm{i}} \tau_{\mathrm{i}}$

$\eta_{2}=n_{\mathrm{i}} T_{\mathrm{i}} \tau_{\mathrm{i}} \frac{\left(\frac{6}{5}\left(\omega_{\mathrm{i}} \tau_{\mathrm{i}}\right)^{2}+2.23\right)}{\left(\omega_{\mathrm{i}} \tau_{\mathrm{i}}\right)^{4}+4.03\left(\omega_{\mathrm{i}} \tau_{\mathrm{i}}\right)^{2}+2.33}$.

The coefficient $\eta_{1}$ is obtained from $\eta_{2}$ by replacing $\omega_{\mathrm{i}}$ by $2 \omega_{\mathrm{i}}$. Here $\omega_{\mathrm{i}}$ is the ion gyrofrequency and $\tau_{\mathrm{i}}$ is the ion collision time, defined for a fully ionized plasma as the inverse of the ion-ion collision frequency:

$\tau_{\mathrm{i}} \equiv \frac{1}{v_{\mathrm{ii}}}=1.03 \times 10^{25} \frac{\sqrt{\mu} T_{\mathrm{i}}^{3 / 2}}{\Lambda Z^{4} n_{\mathrm{i}}}$,

where $\mu=\frac{m_{\mathrm{i}}}{m_{\mathrm{p}}}$ is mass of the ion measured in units of the proton mass, $Z$ is the ion charge number, and $\Lambda$ is the Coulomb logarithm (Spitzer 1962). Throughout this paper we assume an isothermal plasma, i.e. $T_{k}=T, k=\mathrm{e}, \mathrm{i}, \mathrm{n}$.

It is important to remember when using Eqs. (1)-(6) that care must be taken over the plasma $\beta$. As mentioned above for very low plasma $\beta$ the damping of Alfvén waves and fast magnetoacoustic waves are described by Eqs. (1)-(4) while slow magnetoacoustic waves are damped according to the acoustic formulas Eqs. (5)-(6). However, in the high plasma $\beta$ limit both Alfvén and slow magnetoacoustic waves are covered by Eqs. (1)-(2) and fast magnetoacoustic waves are damped according to Eqs. (5)-(6). In all situations the classical sound wave, i.e. parallel propagating pressure perturbations with $k_{\perp}=$ 0 , are covered by Eq. (6).

\subsection{The case of partially ionized plasma}

The presence of neutral atoms causes the Joule heating to increase because the electrons collide with neutrals as well as with ions, and what is more important, the ions collide with neutrals. Thus the value and form of the electric conductivity coefficients in Eqs. (1), (3), and (5) are different for a partially ionized plasma. The expressions for the viscous damping decrements in a partially ionized plasma, $\tilde{\delta}_{\text {visc }}$, should contain now the viscosity coefficients $\tilde{\eta}_{0}, \tilde{\eta}_{1}$, and $\tilde{\eta}_{2}$ modified appropriately to include the neutral gas, also the total density $\rho_{0}$ should include the density of the neutral component as well. Note that throughout this paper variables with a tilda refer to expression which relate to partially ionised plasmas. In a weakly ionized plasma the viscosity coefficients are isotropic and the estimation $\tilde{\eta}_{0,1,2} \sim n_{\mathrm{n}} T \tau_{\mathrm{n}}$ can be applied, where $\tau_{\mathrm{n}}=\left(v_{\mathrm{ni}}+v_{\mathrm{nn}}\right)^{-1}$ is the neutral particle collision time. When the ionization ratio $n_{\mathrm{i}} / n_{\mathrm{n}}$ is sufficiently high the ion viscosity effects will still dominate over the neutral contribution. However, in general the ion collision time in Eqs. (7) should take account of the ion-neutral collisions, i.e. $\tau_{\mathrm{i}}$ should be replaced in Eqs. (7) by $\tilde{\tau}_{\mathrm{i}}=\left(v_{\mathrm{ii}}+v_{\mathrm{in}}\right)^{-1}$.
Either from the generalized Ohm's Law in a partially ionized plasma (Cowling 1957; Zaitsev \& Stepanov 1992), or from direct calculation involving the frictional coefficients of each species (Braginskii 1965), the expression for the frictional heating can be shown to contain, along with the Joule heating, an additional term arising due to the plasma-neutral gas collisional friction. It has the following form (Braginskii 1965):

$Q_{\text {frict }}=\frac{j^{2}}{\sigma}+\frac{1}{\alpha_{\mathrm{n}}}\left(\frac{\xi_{\mathrm{n}}}{c}[\boldsymbol{j} \times \boldsymbol{B}]-\boldsymbol{G}\right)^{2}$,

where

$\boldsymbol{G}=\xi_{\mathrm{n}} \nabla\left(p_{\mathrm{e}}+p_{\mathrm{i}}\right)-\xi_{\mathrm{i}} \nabla p_{\mathrm{n}}$,

$\sigma=\frac{n_{\mathrm{e}} e^{2}}{m_{\mathrm{e}}\left(v_{\mathrm{ei}}^{\prime}+v_{\mathrm{en}}^{\prime}\right)}$ and $\alpha_{\mathrm{n}}=m_{\mathrm{e}} n_{\mathrm{e}} v_{\mathrm{en}}^{\prime}+m_{\mathrm{i}} n_{\mathrm{i}} v_{\mathrm{in}}^{\prime}$. Here $n_{k}$ and $p_{k}, k=\mathrm{e}, \mathrm{i}, \mathrm{n}$ are the number densities and kinetic pressures of each species respectively; $\xi_{\mathrm{n}}=\frac{m_{\mathrm{n}} n_{\mathrm{n}}}{m_{\mathrm{n}} n_{\mathrm{n}}+m_{\mathrm{i}} n_{\mathrm{i}}}$ and $\xi_{\mathrm{i}}=\frac{m_{\mathrm{i}} n_{\mathrm{i}}}{m_{\mathrm{n}} n_{\mathrm{n}}+m_{\mathrm{i}} n_{\mathrm{i}}}$ are the relative densities of neutrals and ions; $v_{\mathrm{ei}}^{\prime}, v_{\mathrm{en}}^{\prime}$ and $v_{\mathrm{in}}^{\prime}$ are the effective electron-ion, electronneutral and ion-neutral collisional frequencies defined as $v_{k l}^{\prime}=$ $\frac{m_{l}}{m_{l}+m_{k}} v_{k l}, \quad k=\mathrm{e}, \mathrm{i}, l=\mathrm{i}, \mathrm{n}$. For estimation of the collision frequencies $v_{\mathrm{ei}}, v_{\mathrm{en}}$ and $v_{\text {in }}$ we use the following expressions:

$v_{\mathrm{ei}}=5.89 \times 10^{-24} \frac{n_{\mathrm{i}} \Lambda Z^{2}}{T^{3 / 2}}$,
$v_{\mathrm{en}}=n_{\mathrm{n}} \sqrt{\frac{8 T}{\pi m_{\mathrm{en}}}} \Sigma_{\mathrm{en}}$,
$v_{\mathrm{in}}=n_{\mathrm{n}} \sqrt{\frac{8 T}{\pi m_{\mathrm{in}}}} \Sigma_{\mathrm{in}}$,

where $\Sigma_{\text {en }} \sim 10^{-15} \mathrm{~cm}^{2}, \Sigma_{\text {in }} \sim 5 \times 10^{-15} \mathrm{~cm}^{2}$ are electronneutral and ion-neutral collisional cross-sections, and $m_{k \mathrm{n}}=$ $\frac{m_{k} m_{\mathrm{n}}}{m_{k}+m_{\mathrm{n}}}, k=\mathrm{e}, \mathrm{i}$.

For an Alfvén wave $\boldsymbol{G}=0$, while for a fast magnetoacoustic wave the ratio of $\boldsymbol{G}$ to the first term in brackets in the Eq. (9) is of the order of $\xi_{\mathrm{i}} C_{\mathrm{s}}^{2} / V_{\mathrm{A}}^{2}$. Neglecting $\boldsymbol{G}$ for both these modes we can again present the frictional heating term (9) as Joule heating:

$Q_{\text {frict }}=\tilde{Q}_{\text {Joule }}=\frac{j_{\|}^{2}}{\sigma}+\frac{j_{\perp}^{2}}{\sigma_{\mathrm{C}}}$,

where $\sigma_{\mathrm{C}}=\frac{\sigma}{1+\frac{\xi_{\mathrm{n}}^{2} B_{0}^{2}}{\alpha_{\mathrm{n}}{ }^{2}} \sigma}$ is the well known Cowling conductivity defined mainly by the ion-neutral collisions. In a strong enough magnetic field $\frac{\sigma}{\sigma_{\mathrm{C}}}=1+\xi_{\mathrm{n}}^{2} \frac{B_{0}^{2} \sigma}{\alpha_{\mathrm{n}} c^{2}} \approx 1+\xi_{\mathrm{n}}^{2} \frac{\omega_{\mathrm{e}} \omega_{\mathrm{i}}}{\max \left\{v_{\mathrm{ei}}^{\prime}, v_{\mathrm{en}}^{\prime}\right\} v_{\mathrm{in}}^{\prime}} \gg 1$. Thus, the Cowling conductivity $\sigma_{\mathrm{C}}$ is much less than the usual conductivity $\sigma$. This fact causes, assuming $([\boldsymbol{j} \times \boldsymbol{B}] \neq 0)$, an increase in the Joule heating in a partially ionized plasma. The significant increase in Joule energy dissipation, due to ionneutral collisions, has been considered in (Piddington 1954; Frank-Kamenetskii 1961) and applied to solar phenomena in (Zaitsev \& Stepanov 1992; Khodachenko \& Zaitsev 1992; Khodachenko 1996). 
Based on Eq. (12), the expressions (1) and (3) can be rewritten for logarithmic damping decrements, $\tilde{\delta}_{\text {Joule }}^{\text {A.w. }}, \tilde{\delta}_{\text {Joule }}^{\text {f.ms.w. }}$, of the Alfvén and fast magnetoacoustic waves in a partially ionized plasma as the following:

$$
\begin{aligned}
2 \omega \tilde{\delta}_{\text {Joule }}^{\text {A.w. }} & \equiv \frac{1}{\tilde{\tau}_{\text {Joule }}^{\text {A.w. }}}=\frac{c^{2}}{4 \pi \sigma} k_{\perp}^{2}+\frac{c^{2}}{4 \pi \sigma_{\mathrm{C}}} k_{\|}^{2} \\
& =\frac{c^{2}}{4 \pi \sigma} k^{2}+\frac{c^{2}}{4 \pi} \frac{\xi_{\mathrm{n}}^{2} B_{0}^{2}}{\alpha_{\mathrm{n}} c^{2}} k_{\|}^{2}, \\
2 \omega \tilde{\delta}_{\text {Joule }}^{\text {f.ms.w. }} & \equiv \frac{1}{\tilde{\tau}_{\text {Joule }}^{\text {f.ms.w. }}}=\frac{c^{2}}{4 \pi \sigma_{\mathrm{C}}} k^{2} \\
& =\frac{c^{2}}{4 \pi \sigma} k^{2}+\frac{c^{2}}{4 \pi} \frac{\xi_{\mathrm{n}}^{2} B_{0}^{2}}{\alpha_{\mathrm{n}} c^{2}} k^{2} .
\end{aligned}
$$

For the acoustic wave both terms in brackets in the Eq. (9) are of the same order and should be taken into account. In particular, based on Eqs. (9) and (10), and applying techniques described in Braginskii (1965), one will obtain for the case of $m_{\mathrm{i}}=m_{\mathrm{n}}$ :

$$
\begin{aligned}
2 \omega \tilde{\delta}_{\text {frict }}^{\text {s.w. }} \equiv & \frac{1}{\tilde{\tau}_{\text {frict }}^{\text {s.w. }}}=\frac{c^{2}}{4 \pi \sigma} \frac{C_{\mathrm{s}}^{2}}{V_{\mathrm{A}}^{2}} k_{\perp}^{2} \\
& +\frac{\xi_{\mathrm{n}}^{2} C_{\mathrm{s}}^{2} \rho_{0}}{\alpha_{\mathrm{n}}}\left(k_{\|}^{2} \frac{n_{\mathrm{e}}^{2}}{n_{0}^{2}}+k_{\perp}^{2} \frac{\left(n_{\mathrm{i}}+n_{\mathrm{n}}\right)^{2}}{n_{0}^{2}}\right) .
\end{aligned}
$$

Here $n_{0}=\sum_{k} n_{k}, k=\mathrm{i}$, e, n, and the total density, $\rho_{0}$, of partially ionized plasma includes now as the density of the neutral gas. Note that the pressure function $\boldsymbol{G}$ in the Eq. (9) results in appearance of the coefficient $\frac{\left(n_{\mathrm{i}}+n_{\mathrm{n}}\right)^{2}}{n_{0}^{2}}$ near $k_{\perp}^{2}$ in brackets in the Eq. (15), as well as causes the term proportional to $k_{\|}^{2}$ there.

Therefore, in a partially ionized plasma the expression for the acoustic wave frictional damping decrement (15) contains additional terms, as compared to the case of the fully ionized plasma (5). It is important to note that the frictional damping of acoustic waves takes place in a partially ionized plasma not only for waves propagating across the background magnetic field $\left(k_{\perp} \neq 0\right)$, as it was in the fully ionized plasma, but also for waves propagating along the magnetic field $\left(k_{\perp}=0, k_{\|} \neq\right.$ 0 ). The additional terms (in brackets) in Eq. (15) are usually greater than the first term. In particular, for waves propagating across the magnetic field $\left(k_{\perp} \neq 0, \quad k_{\|}=0\right)$ the ratio of the first term in (15) to the second is $\frac{n_{0}^{2}}{\left(n_{\mathrm{i}}+n_{\mathrm{n}}\right)^{2}} \frac{\sigma_{\mathrm{C}}}{\sigma} \ll 1$. This means that in a partially ionized plasma the frictional damping of acoustic waves is much stronger than it is in a fully ionized plasma.

\section{Application to the Sun}

As related to the phenomena on the Sun, the case of longitudinal with respect to the magnetic field $\left(k_{\perp}=0, \quad k_{\|} \neq 0\right.$ ), propagation of MHD waves appears to be the most important. These waves are believed to be guided within magnetic flux tubes, which therefore play the role of channels providing the energetic coupling of different layers of the solar atmosphere.

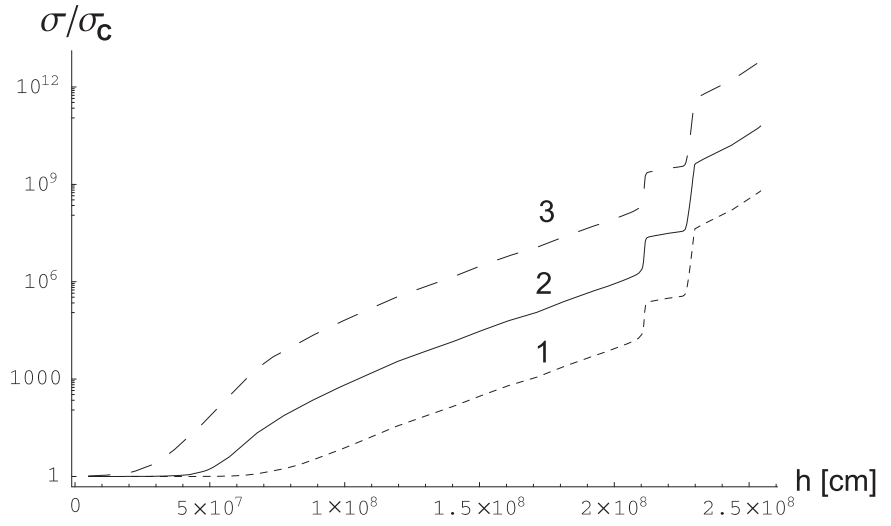

Fig. 1. Variation of $\frac{\sigma}{\sigma_{\mathrm{C}}}$ with height for the quiet Sun model of Vernazza et al. (1981) for different $\left.B_{0}: 1\right) 10 \mathrm{G}$; 2) $100 \mathrm{G}$ and 3) $1000 \mathrm{G}$.

From Eqs. (1), (3) and (13), (14), parallel propagating Alfvén and fast magnetoacoustic waves are equally damped due to the collisional friction (Joule) dissipation. But because of $\frac{\sigma}{\sigma_{\mathrm{C}}} \gg 1$, this damping is much higher for the partially ionized plasma of the solar photosphere, chromosphere and prominences, in comparison to the coronal plasma. Figure 1 shows the variation of the ratio $\frac{\sigma}{\sigma_{\mathrm{C}}}$ with height in the low solar atmosphere (photosphere/chromosphere) calculated for different values of the magnetic field $B_{0}$. For this calculation we used the solar atmospheric plasma parameters provided by the VAL C model of the quiet Sun (Vernazza et al. 1981).

Parallel propagating acoustic waves are not damped due to friction in a fully ionized coronal plasma (see Eq. (5)), but are damped in the partially ionized solar photospheric, chromospheric and prominence plasmas (see Eq. (15)).

Viscous damping of longitudinally propagating $\left(k_{\perp}=\right.$ $0, k_{\|} \neq 0$ ) Alfvén and fast magnetoacoustic waves again are described by the same expression, which follows from (2) and (4). Different viscous damping of MHD waves in different parts of the solar atmosphere appears due to the corresponding changes of the plasma density and viscosity coefficients in accordance with varying solar photospheric/chromospheric/coronal plasma parameters.

Before further presentation of our results regarding MHD waves damping in the solar partially ionized plasmas we would like to make a remark concerning the difference between the present work and existing similar studies. The previous investigations were usually addressed to particular regions of the solar atmosphere. For example photosphere/chromosphere in DePontieu et al. (2001) and the chromosphere in Goodman $(2000,2001)$. Other works concentrated on a particular MHD mode, e.g. Alfven waves in DePontieu et al. (2001), and James \& Erdélyi (2002) or slow magnetoacoustic waves in Goodman $(2000,2001))$. In the present paper we do not limit ourselves to consideration of a particular mode, or particular region. We perform our analysis in the whole range of the solar partially ionized plasmas, including the photosphere, chromosphere and material of prominences, as well as consider all the important solar MHD modes: Alfvén wave, 
fast magnetoacoustic wave and slow magnetoacoustic/acoustic wave. One more important difference of our work from others consists in its main goal: to perform a quantitative comparison of the efficiency of MHD waves damping due to collisional friction and viscous energy dissipation mechanisms in the partially ionized solar plasmas. In the previous studies one of the above damping mechanisms was usually neglected. The reasoning for this was given in a brief semi-quantitative estimative style, like for example in the paper by Goodman (2000). The quantitative study of this subject has never been done for the solar atmosphere and we try to fill this gap. It is also important to note that, considered here, collisional friction energy dissipation (i.e. heat generated in a gas due to particle collisions) in a partially ionized plasma represents a more general case than just the resistive (Joule) energy dissipation (see Eq. (9)). Only in the case of certain MHD modes, when disturbances of the pressure are absent or can be neglected, can the collisional friction energy dissipation rate be represented as a resistive dissipation (see Eq. (12)), i.e. expressed, similar to the fully ionized plasma case, in terms of electric conductivities. This is, in particular, not the case of the acoustic wave, considered among others below.

\subsection{MHD wave damping in the solar photosphere/chromosphere}

Based on Eqs. (2), (4), (13), (14) and taking into account the corresponding estimations for the viscosity coefficients, we compare the collisional friction (Joule) and viscous damping times of Alfvén and fast magnetoacoustic waves propagating along the magnetic field $(\|)$ in the partially ionized plasmas of the low solar atmosphere:

$\left.\frac{\tilde{\tau}_{\text {Joule }}}{\tilde{\tau}_{\text {visc }}}\right|_{\substack{\text { A.w.w.(II) } \\ \text { f.II) }}}=\frac{4 \pi \tilde{\eta}_{2} \sigma_{\mathrm{C}}}{c^{2} \rho_{0}}$.

In the photospheric case of a weakly ionized plasma $\left(\tilde{\eta}_{2} \sim\right.$ $\left.n_{\mathrm{n}} T \tau_{\mathrm{n}}\right)$ and strong magnetic field $\left(\frac{\omega_{\mathrm{e}} \omega_{\mathrm{i}}}{v_{\mathrm{en}}^{\prime} v_{\mathrm{in}}^{\prime}} \gg 1\right)$, Eq. (16) gives $\left.\frac{\tilde{\tau}_{\text {Joule }}}{\tilde{\tau}_{\text {visc }}}\right|_{\substack{\text { A.w.(II) } \\ \text { f.ms.w.(I) }}} \approx \frac{\mu}{1+\mu} \frac{\mu+\left(n_{\mathrm{n}} / n\right)}{1+\left(n_{\mathrm{n}} / n\right)} \beta_{\mathrm{i}} \ll 1$, where $\beta_{\mathrm{i}}=\frac{4 \pi T n}{B_{0}^{2}} \ll 1$. Here and below we assume $n_{\mathrm{e}}=n_{\mathrm{i}}=n$.

In Fig. 2 the dependence of $\left.\frac{\tilde{\tau}_{\text {Joule }}}{\tilde{\tau}_{\text {visc }}}\right|_{\substack{\text { f.w. .(I) } \\ \text { f.m.w.(I) }}}$ on height in the low solar atmosphere, calculated for the VAL C model parameters (Vernazza et al. 1981) and different values of the magnetic field $B_{0}$, is presented. As can be seen in Fig. 2, the effects of frictional (Joule) energy dissipation, caused mainly by the ion-neutral collisions, are much stronger for the damping of Alfvén and fast magnetoacoustic waves in the low solar atmosphere than viscosity. Therefore, the correct description of the propagation of these waves in the partially ionized solar photospheric and chromospheric plasmas requires the inclusion of collisional energy dissipation mechanisms, whereas the viscous energy dissipation can be quite often neglected (DePontieu \& Haerendel 1998; DePontieu et al. 2001).

Simply modifying of the magnetic diffusion coefficient in the induction equation is not sufficient to correctly account for

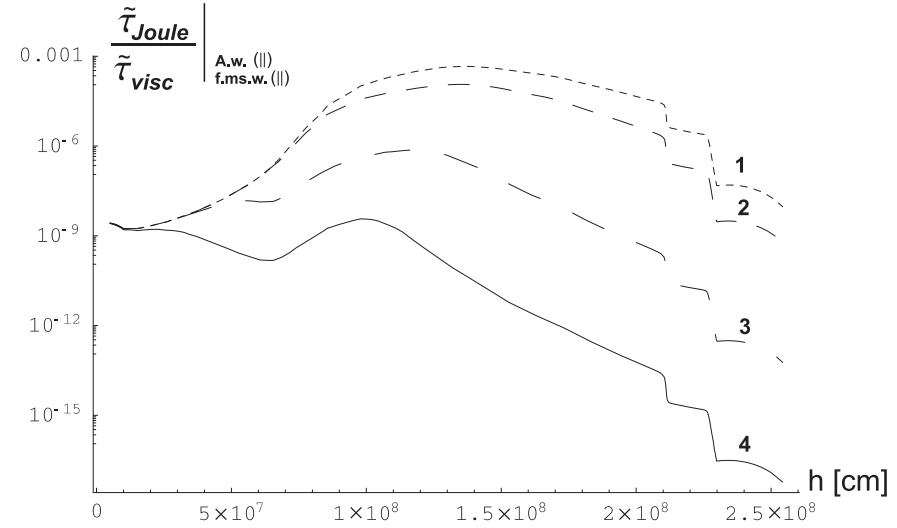

Fig. 2. Variation of $\left.\frac{\tilde{\tau}_{\text {Joule }}}{\tilde{\tau}_{\text {visc }}}\right|_{\text {A.w.(I) }}$ with height in the low solar atmosphere (Vernazza et al. 1981) for different $\left.B_{0}: 1\right) 5 \mathrm{G} ; 2$ ) $10 \mathrm{G}$; 3) $100 \mathrm{G}$; and 4) $1000 \mathrm{G}$.

all the resistive effects in a partially ionized plasma. The generalized Ohm's Law is more complicated than just $\boldsymbol{E}+\frac{1}{c}[\boldsymbol{V} \times$ $\boldsymbol{B}]=\frac{\boldsymbol{j}}{\sigma}$ (Bakhareva et al. 1992; Khodachenko \& Zaitsev 1992; 2002). The heating term $\left(\boldsymbol{E}+\frac{1}{c}[\boldsymbol{V} \times \boldsymbol{B}]\right) \cdot \boldsymbol{j}$ in the energy equation also becomes more complicated, and instead of just $j^{2} / \sigma$ includes other terms, among which the most significant is the term $j_{\perp}^{2} / \sigma_{\mathrm{C}} \gg j^{2} / \sigma$. Thus simply increasing the magnetic diffusion will drive the magnetic field towards a potential field while the generalized Ohm's Law has an enhanced perpendicular Cowling resistivity which would leave any non-linear force free field unaffected. Since, as shown below, the Cowling conductivity term is dominant in regions of the lower atmosphere this is especially important as a large scalar resistivity will quickly, and erroneously, establish a potential field instead of leaving any parallel current unchanged.

Next we estimate the frictional damping of acoustic waves in a partially ionized plasma. This effect could be important, in particular, for acoustic waves propagating in the low solar atmosphere. Based on Eqs. (15) and (6), we compare the frictional and viscous damping times of the acoustic wave propagating along the magnetic field $(\|)$ :

$\left.\frac{\tilde{\tau}_{\text {frict }}}{\tilde{\tau}_{\text {visc }}}\right|_{\text {s.w.(II) }}=\frac{4}{3} \frac{\tilde{\eta}_{0} \alpha_{\mathrm{n}}}{\xi_{\mathrm{n}}^{2} C_{\mathrm{s}}^{2} \rho_{0}^{2}} \frac{n_{0}^{2}}{n^{2}}$.

Using the above definitions for $\tilde{\eta}_{0}, \alpha_{\mathrm{n}}$ and $C_{\mathrm{s}}^{2}$ the ratio (17) can be written as

$\left.\frac{\tilde{\tau}_{\text {frict }}}{\tilde{\tau}_{\text {visc }}}\right|_{\text {s.w.(I) }}=\frac{0.64}{\gamma} \frac{\tilde{\tau}_{\mathrm{i}} v_{\mathrm{in}}}{\xi_{\mathrm{n}}^{2}} \frac{\left(2+n_{\mathrm{n}} / n\right)}{\left(1+n_{\mathrm{n}} / n\right)}$.

Here we take into account the fact that Eq. (15), used for the derivation of Eq. (17), was obtained with the assumption $m_{\mathrm{i}}=$ $m_{\mathrm{n}}$.

When the ionization degree is high, i.e. $n_{\mathrm{n}} \rightarrow 0$, Eq. (18) gives $\left.\frac{\tilde{\tau}_{\text {frict }}}{\tilde{\tau}_{\text {visc }}}\right|_{\text {s.w.(\|) }} \propto \frac{1}{n_{\mathrm{n}}} \longrightarrow \infty$. Thus, the role of the acoustic wave frictional damping in this case is small compared to the viscous damping. Contrary to that, for a weakly ionized plasma 


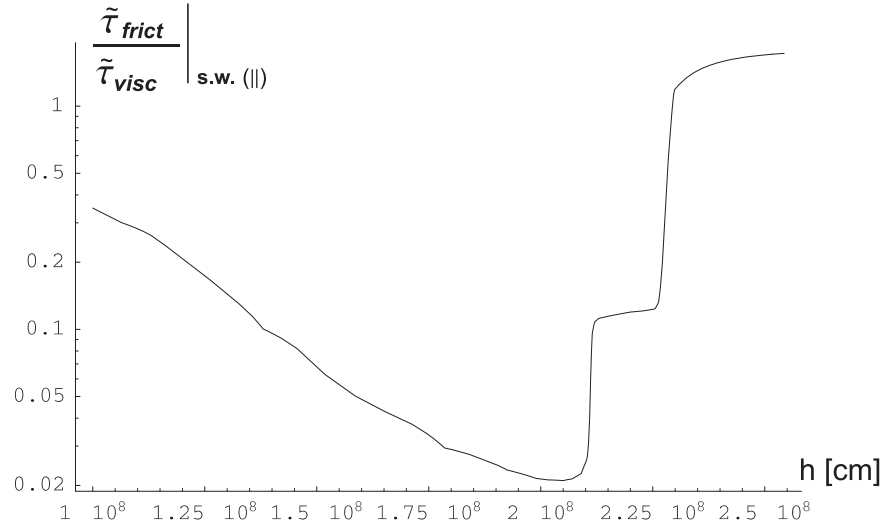

Fig. 3. Variation of $\left.\frac{\tilde{\tau}_{\text {frict }}}{\tilde{\tau}_{\text {visc }}}\right|_{\text {s.w.(II) }}$ with height in the solar chromosphere (Vernazza et al. 1981).

$\left(n_{\mathrm{n}} / n \rightarrow \infty\right)$, when one can assume $\tilde{\tau}_{\mathrm{i}} \approx v_{\mathrm{in}}^{-1}$, it follows from Eq. (18) that $\left.\frac{\tilde{\tau}_{\text {frict }}}{\tilde{\tau}_{\text {visc }}}\right|_{\text {s.w.(\|) }} \longrightarrow 0.64 / \gamma<1$. Therefore the collisional energy dissipation mechanism can dominate over the viscous one for the acoustic wave damping in a partially ionized plasma.

Variation with height in the solar chromosphere of the ratio $\left.\frac{\tilde{\tau}_{\text {frict }}}{\tilde{\tau}_{\text {visc }}}\right|_{\text {s.w.(\|) }}$ defined by Eq. (17) is presented in Fig. 3. Here we again used the solar atmospheric plasma parameters provided by the VAL C model of the quiet Sun (Vernazza et al. 1981), and limit our consideration to the height interval of the middle and upper chromosphere, where the ionized hydrogen provides the majority of ions so that the case $m_{\mathrm{i}}=m_{\mathrm{n}}$, for which Eqs. (15) and (17) are valid, is more or less realized. As can be seen from Fig. 3, the frictional damping of the acoustic wave in the low chromosphere is stronger than the viscous damping. At the same time, above the middle chromosphere the efficiency of both damping mechanisms becomes to be of the same order of magnitude. Thus none of the mechanisms can be neglected in models.

\subsection{MHD wave damping in the solar corona and prominences}

The majority of solar coronal plasma can be considered as a fully ionized medium and the effects of the small amount of neutral atoms are usually ignored. But even in this case, the collisional friction (Joule) damping of MHD waves under certain conditions could be comparable or even stronger than the viscous damping. The ratio of the resistive and viscous damping times of Alfvén and fast magnetoacoustic waves, propagating along the magnetic field in the corona can be easily obtained from Eqs. (1)-(4). It has the same form as Eq. (16), where the ion viscosity $\eta_{2}$ and transverse conductivity of a fully ionized plasma, $\sigma_{\perp}$, should be substituted in place of $\tilde{\eta}_{2}$ and $\sigma_{\mathrm{C}}$ respectively. In the case of a strong magnetic field, $\omega_{\mathrm{i}} \tau_{\mathrm{i}} \gg 1$ (this condition is well satisfied in the solar corona for $B_{0}>10 \mathrm{G}$ ), taking into account Eqs. (7), (11) and the expression for the
Table 1. $\left.\frac{\tau_{\text {Joule }}}{\tau_{\text {visc }}}\right|_{\substack{\text { f.w...(I) } \\ \text { f.(I) }}}$ calculated for $B_{0}=10 ; 50 ; 100 \mathrm{G}$ in the corona.

\begin{tabular}{cccc}
\hline \hline & $T=10^{5} \mathrm{~K}$ & $T=5 \times 10^{5} \mathrm{~K}$ & $T=10^{6} \mathrm{~K}$ \\
\hline \multirow{4}{*}{$n=10^{8} \mathrm{~cm}^{-3}$} & 0.0063 & 0.0315 & 0.063 \\
& 0.000252 & 0.00126 & 0.00252 \\
& 0.000063 & 0.000315 & 0.00063 \\
\hline \multirow{3}{*}{$n=10^{10} \mathrm{~cm}^{-3}$} & 0.63 & 3.15 & 6.3 \\
& 0.0252 & 0.126 & 0.252 \\
& 0.0063 & 0.0315 & 0.063 \\
$n=10^{12} \mathrm{~cm}^{-3}$ & 63 & 315 & 630 \\
& 2.52 & 12.6 & 25.2 \\
& 0.63 & 3.15 & 6.3 \\
\hline
\end{tabular}

transverse conductivity $\sigma_{\perp}=\frac{n e^{2}}{m_{\mathrm{e}} v_{\mathrm{ei}}^{\prime}}$, provided by Braginskii (1965), after substitution of all numeric constants the ratio of the damping times is:

$$
\begin{aligned}
\left.\frac{\tau_{\text {Joule }}}{\tau_{\text {visc }}}\right|_{\substack{\text { f.w. } .(\|) .(I) \\
\text { f.ms.. }}} & =18.17 \beta \sqrt{\mu} \\
& \approx 6.3 \times 10^{-14} \sqrt{\mu} \frac{n T\left[\mathrm{~K}^{\circ}\right]}{B_{0}^{2}} .
\end{aligned}
$$

For convenience of application to the Sun the temperature in Eq. (19) is expressed in Kelvins. It follows from Eq. (19), for sufficiently small plasma $\beta$ the resistive damping of Alfvén and fast magnetoacoustic waves in the corona can be stronger, or comparable with the viscous damping, and therefore, should not be neglected. The range of possible values of $\left.\frac{\tau_{\text {Joule }}}{\tau_{\text {visc }}}\right|_{\substack{\text { A.w.(II) } \\ \text { f.ms..(I) }}}$ calculated for some typical coronal plasma parameters and different values of the magnetic field is presented in the Table 1. For each particular density and temperature of plasma we give in the Table 1 three values of $\left.\frac{\tau_{\text {Joule }}}{\tau_{\text {visc }}}\right|_{\substack{\text { A.w.(II). } \\ \text { fli) }}}$. The upper of these values corresponds to $B_{0}=10 \mathrm{G}$, the middle, to $B_{0}=50 \mathrm{G}$, and the bottom, to $B_{0}=100 \mathrm{G}$. All of these numbers are simply classical results with no neutral component.

Returning to partially ionised plasmas specific examples where the neutral fraction is significant are of course prominences. This is a relatively cold $\left(T=(6-10) \times 10^{3} \mathrm{~K}\right)$ and dense $\left(n=(1-50) \times 10^{10} \mathrm{~cm}^{-3}\right)$ partially ionized $\left(\frac{n_{\mathrm{n}}}{n}=0.05-1\right)$, magnetized $(B \sim 10 \mathrm{G})$ medium, with the majority of ions provided by the ionized hydrogen (i.e. $\mu \approx 1$ ).

The ratios of collisional friction (Joule) and viscous damping times of the Alfvén, fast magnetoacoustic and acoustic waves propagating along the magnetic field in the prominence plasma are defined in general by Eqs. (16) and (17). From these, Eq. (18) for the acoustic wave, derived from Eq. (17) can be directly applied for the case of prominences. It yields $\left.\frac{\tilde{\tau}_{\text {frict }}}{\tilde{\tau}_{\text {visc }}}\right|_{\text {s.w.(I) }}=5 \times 10^{-3}-10^{-2}$ for the prominence parameters given above. 

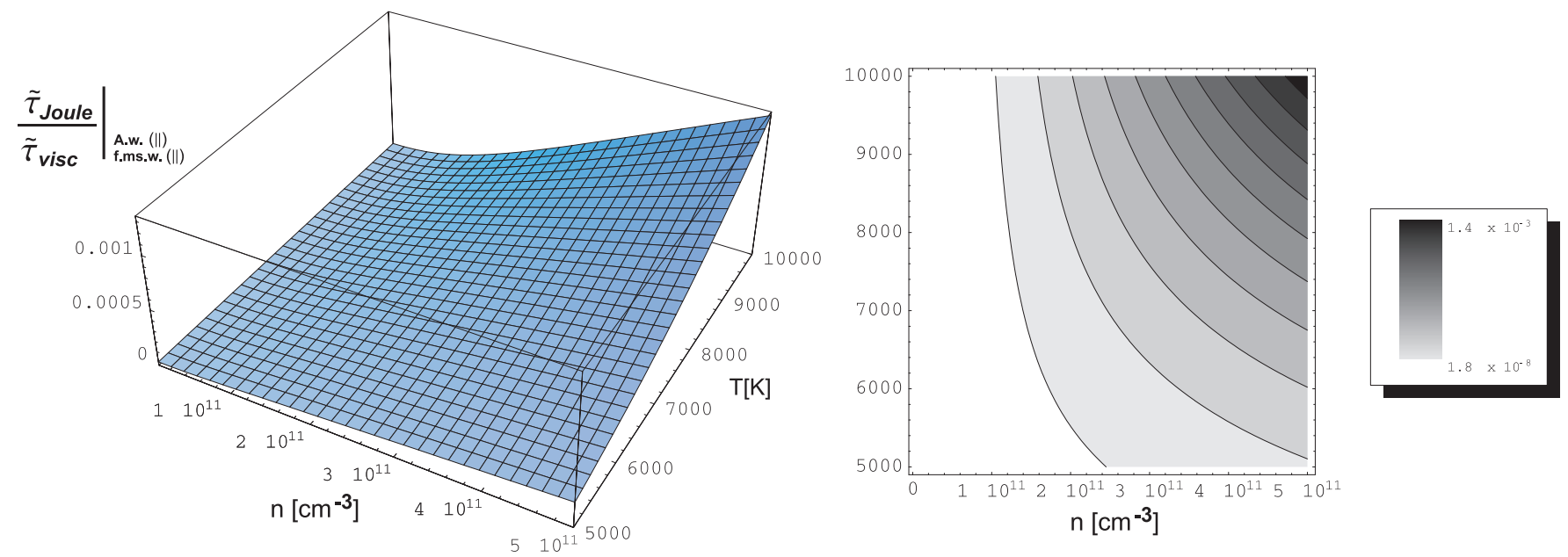

a.
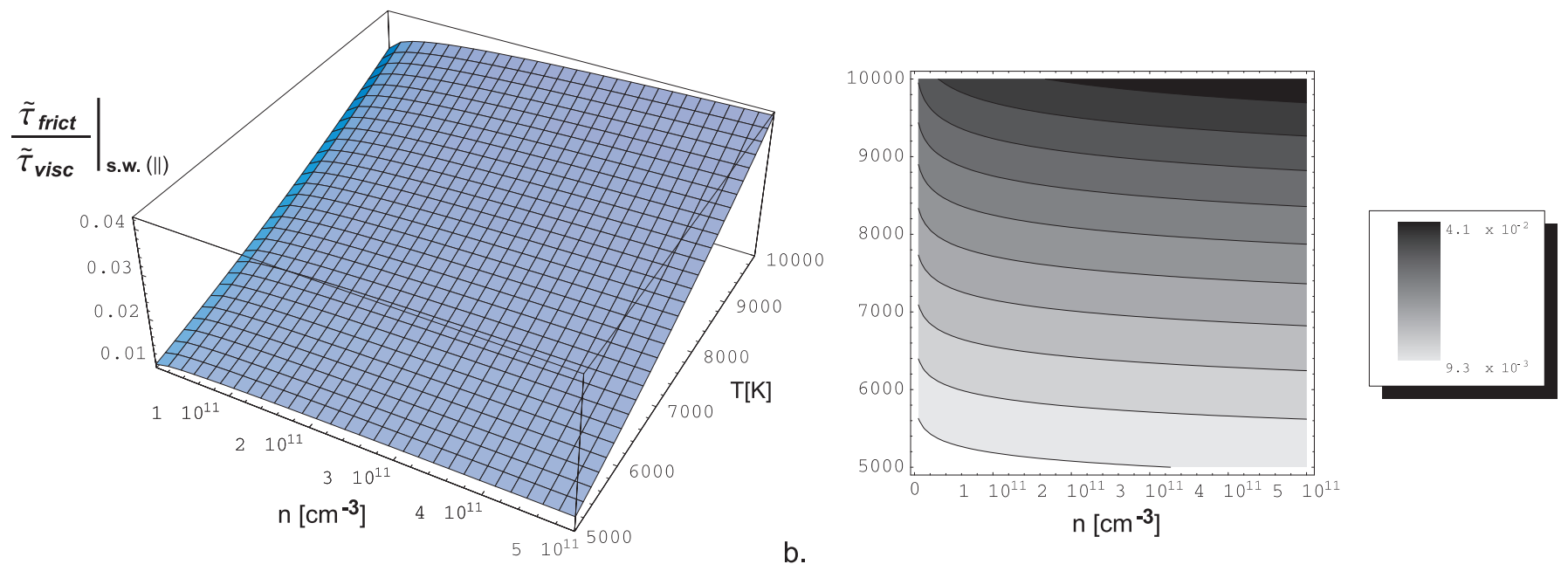

Fig. 4. Typical values of the collisional and viscous damping time ratios for parallel propagating a) Alfvén and fast magnetoacoustic waves $\left.\frac{\tilde{\tau}_{\text {Joule }}}{\tilde{\tau}_{\text {visc }}}\right|_{\substack{\text { A.w..(II) } \\ \text { f.m...(I) }}}$ and b) acoustic wave $\left.\frac{\tilde{\tau}_{\text {frict }}}{\tilde{\tau}_{\text {visc }}}\right|_{\text {s.w.(II) }}$ in the prominence plasma for $B_{0}=10 \mathrm{G}$ and $\frac{n_{\mathrm{n}}}{n}=1$.

For not very dense prominences $\left(n=(1-3) \times 10^{10} \mathrm{~cm}^{-3}\right)$ the conditions of a strong magnetic field

$\frac{\omega_{\mathrm{e}} \omega_{\mathrm{i}}}{\max \left\{v_{\mathrm{en}}^{\prime}, v_{\mathrm{ei}}^{\prime}\right\} v_{\mathrm{in}}^{\prime}}=(4-100) \times 10^{5} \gg 1$

$\omega_{\mathrm{i}} \tilde{\tau}_{\mathrm{i}}=14-3>1$

are usually satisfied. In this case Eq. (16) can be transformed to:

$$
\begin{aligned}
&\left.\frac{\tilde{\tau}_{\text {Joule }}}{\tilde{\tau}_{\text {visc }}}\right|_{\substack{\text { A.w.(II) } \\
\text { f.ms..(I) }}}=\frac{4 \pi \tilde{\eta}_{2} \alpha_{\mathrm{n}}}{\rho_{0} \xi_{\mathrm{n}}^{2} B_{0}^{2}} \\
& \approx \frac{3}{5} \frac{\left(1+\left(n_{\mathrm{n}} / n\right)\right)}{\left(n_{\mathrm{n}} / n\right)^{2}} \frac{\nu_{\text {in }}}{\omega_{\mathrm{i}}}\left(\omega_{\mathrm{i}} \tilde{\tau}_{\mathrm{i}}\right)^{-1} \beta_{\mathrm{i}} \ll 1,
\end{aligned}
$$

which again indicates the dominant role of the frictional wave damping mechanism over viscosity.

An overview of the damping time ratios defined by Eqs. (16) and (17) for the range of plasma densities and temperatures typical for prominences, is presented in Fig. 4. We have assumed $B_{0}=10 \mathrm{G}$ and $\frac{n_{\mathrm{n}}}{n}=1$ in these calculations. A decrease of $\frac{n_{\mathrm{n}}}{n}$ results in a slight increase of $\left.\frac{\tilde{\tau}_{\text {Joule }}}{\tilde{\tau}_{\text {visc }}}\right|_{\substack{\text { A.w...(I) } \\ \text { f.m...(I) }}}$ and $\left.\frac{\tilde{\tau}_{\text {frict }}}{\tilde{\tau}_{\text {visc }}}\right|_{\text {s.w.(II) }}$. As can be seen from Fig. 4, for the Alfvén, fast magnetoacoustic and acoustic waves propagating along the magnetic field in the prominence plasma, the collisional friction energy dissipation mechanism is more efficient than the viscosity, which can be safely neglected in the majority of cases.

Of interest for prominences is also the transverse $\left(k_{\perp} \neq\right.$ $0, k_{\|}=0$ ) propagation of MHD waves with respect to the background magnetic field. Based on Eqs. (4), (6) (with partially ionized plasma viscosity coefficients) and Eqs. (14), (15) we define the ratios of collisional friction (Joule) and viscous damping times of fast magnetoacoustic and acoustic waves propagating across the magnetic field $(\perp)$, and compare them with the corresponding ratios (Eqs. (16) and (17)) in the case of longitudinal wave propagation:

$$
\begin{aligned}
\left.\frac{\tilde{\tau}_{\text {Joule }}}{\tilde{\tau}_{\text {visc }}}\right|_{\text {f.ms.w.( } \perp)} & =\frac{4 \pi\left(\tilde{\eta}_{0} / 3+\tilde{\eta}_{1}\right) \sigma_{\mathrm{C}}}{c^{2} \rho_{0}} \\
& =\left.\frac{\tilde{\eta}_{0} / 3+\tilde{\eta}_{1}}{\tilde{\eta}_{2}} \frac{\tilde{\tau}_{\text {Joule }}}{\tilde{\tau}_{\text {visc }}}\right|_{\text {f.ms.w.(\|) }},
\end{aligned}
$$



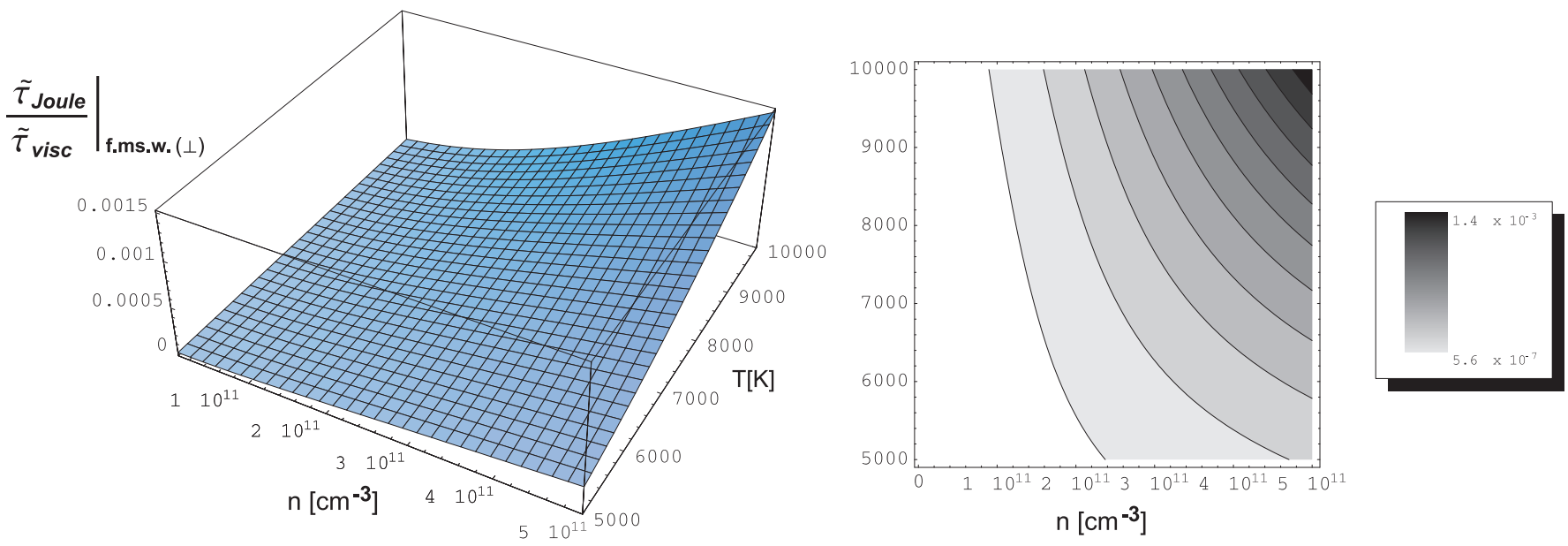

a.
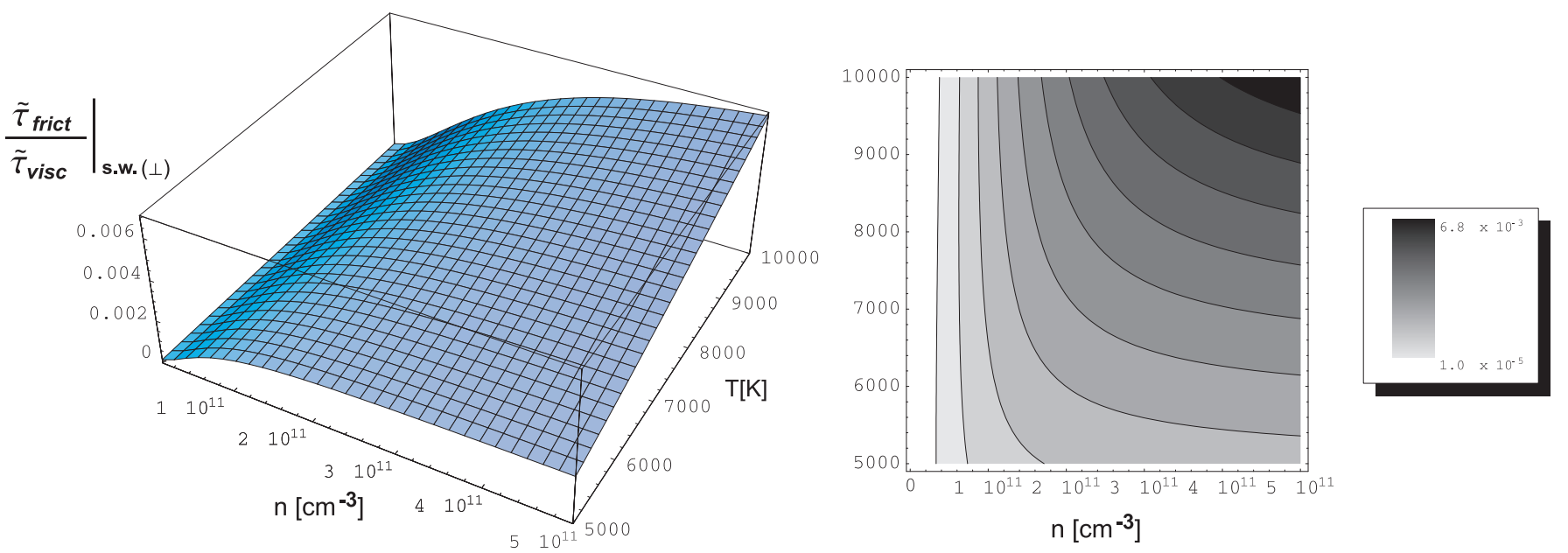

b.

Fig. 5. Typical values of the collisional and viscous damping time ratios for propagating across the magnetic field a) fast magnetoacoustic $\left.\frac{\tilde{\tau}_{\text {Joule }}}{\tilde{\tau}_{\text {visc }}}\right|_{\text {f.ms.w. }(\perp)}$ and b) acoustic $\left.\frac{\tilde{\tau}_{\text {frict }}}{\tilde{\tau}_{\text {visc }}}\right|_{\text {s.w.( } \perp)}$ wave in the prominence plasma for $B_{0}=10 \mathrm{G}$ and $\frac{n_{\mathrm{n}}}{n}=1$.

$$
\begin{aligned}
\left.\frac{\tilde{\tau}_{\text {frict }}}{\tilde{\tau}_{\text {visc }}}\right|_{\text {s.w.( } \perp)} & \approx \frac{\tilde{\eta}_{2} \alpha_{\mathrm{n}}}{\xi_{\mathrm{n}}^{2} C_{\mathrm{s}}^{2} \rho_{0}^{2}} \frac{n_{0}^{2}}{\left(n+n_{\mathrm{n}}\right)^{2}} \\
& =\left.\frac{3}{4} \frac{\tilde{\eta}_{2}}{\tilde{\eta}_{0}}\left(1+\frac{n_{\mathrm{n}}}{n}\right)^{-2} \frac{\tilde{\tau}_{\text {frict }}}{\tilde{\tau}_{\text {visc }}}\right|_{\text {s.w.(I) }} .
\end{aligned}
$$

The factor $\frac{\tilde{\eta}_{0} / 3+\tilde{\eta}_{1}}{\tilde{\eta}_{2}}$ in Eq. (21) is $>1$ in prominence plasma conditions. Thus, the relative role of the effect of collisional damping of fast magnetoacoustic waves, as compared to the viscosity damping, is smaller for waves propagating across the magnetic field, than for longitudinally propagating waves. In spite of this, however, the collisional damping of this mode, i.e. the case of propagation across the magnetic field, still remains stronger in the prominence plasma than viscous damping. This fact is demonstrated in Fig. 5a, where the ratio of collisional and viscous damping times, defined by Eq. (21) is presented for the range of plasma densities and temperatures typical of prominences. For definiteness we assume in this calculation $B_{0}=10 \mathrm{G}$ and $\frac{n_{\mathrm{n}}}{n}=1$. Similar to the case of the longitudinal propagation of fast magnetoacoustic wave, a decrease of $\frac{n_{\mathrm{n}}}{n}$ results in a slight increase of $\left.\frac{\tilde{\tau}_{\text {Joule }}}{\tilde{\tau}_{\text {visc }}}\right|_{\text {f.ms.w.( } \perp)}$, which nevertheless still remains $<1$ for all reasonable prominence plasma parameters.

As can be seen from Eq. (7), the factor $\frac{3}{4} \frac{\tilde{\eta}_{2}}{\tilde{\eta}_{0}}\left(1+\frac{n_{\mathrm{n}}}{n}\right)^{-2}$ in Eq. (22) always remains $<1$ under prominence plasma conditions. This means that the role of collisional damping of acoustic waves compared to their viscous damping in the case of waves propagation across the magnetic field is even stronger than in the case of longitudinal propagation. The dependence of the damping times ratio $\left.\frac{\tilde{\tau}_{\text {frict }}}{\tilde{\tau}_{\text {visc }}}\right|_{\text {s.w.( } \perp)}$, on the prominence plasma parameters is presented in Fig. $5 b$, where we again assume $B_{0}=10 \mathrm{G}$ and $\frac{n_{\mathrm{n}}}{n}=1$.

The collisional damping of MHD waves in the prominence plasma is always stronger than their viscous damping. So, it should be considered as the main mechanism of the MHD wave 
energy dissipation in prominences. Collisional damping must therefore be included in any attempt to describe the observed oscillatory motion of prominence material.

\section{Generalized Ohm's Law and magnetic induction equations in the low solar atmosphere}

According to estimations performed in this paper, the frictional energy dissipation mechanism in majority of cases appears to be the most important mechanism for MHD wave damping in partially ionized plasmas. For the self-consistent description of the collisional friction damping of MHD waves the generalized Ohm's Law (Cowling 1957; Braginskii 1965; Bakhareva et al. 1992, 1992; Khodachenko \& Zaitsev 1992, 2002)

$$
\begin{aligned}
\boldsymbol{E}+\frac{1}{c}[\boldsymbol{V} \times \boldsymbol{B}]= & \frac{\varepsilon \boldsymbol{G}-\nabla p_{\mathrm{e}}}{e n}+\frac{\boldsymbol{j}}{\sigma}+\frac{\left(1-2 \varepsilon \xi_{\mathrm{n}}\right)}{e n c}[\boldsymbol{j} \times \boldsymbol{B}] \\
& -\frac{\xi_{\mathrm{n}}}{c \alpha_{\mathrm{n}}}\left\{\xi_{\mathrm{n}} \frac{[[\boldsymbol{j} \times \boldsymbol{B}] \times \boldsymbol{B}]}{c}-[\tilde{\boldsymbol{G}} \times \boldsymbol{B}]\right\}
\end{aligned}
$$

should be included into the modelling set of MHD equations. Here $\boldsymbol{V}=\xi_{\mathrm{n}} \boldsymbol{v}_{\mathrm{n}}+\xi_{\mathrm{i}} \boldsymbol{v}_{\mathrm{i}}$ is the velocity of the center of mass of the partially ionized plasma and $\varepsilon=\frac{n_{\mathrm{e}} m_{\mathrm{e}} v_{\mathrm{en}}^{\prime}}{\alpha_{\mathrm{n}}}=\frac{v_{\mathrm{en}}^{\prime}}{v_{\mathrm{en}}^{\prime}+\left(m_{\mathrm{i}} / m_{\mathrm{e}}\right) v_{\mathrm{in}}^{\prime}}$. The pressure function $\boldsymbol{G}$ in Eq. (23) is defined by Eq. (10).

Application of the generalized Ohm's Law (Eq. (23)) changes the form of the magnetic induction equation and the Joule heating term $\left(\boldsymbol{E}+\frac{1}{c}[\boldsymbol{V} \times \boldsymbol{B}]\right) \cdot \boldsymbol{j}$ in the energy equation. In the easiest case of a cold strongly magnetized plasma (plasma $\beta \ll 1$ ) and relatively slow processes, the pressure gradient terms (the pressure function $\boldsymbol{G}$ ) and Hall term in Eq. (23) can be neglected. Thus, the corresponding induction equation will have the following form:

$$
\begin{aligned}
\frac{\partial \boldsymbol{B}}{\partial t}=\nabla \times[\boldsymbol{V} \times \boldsymbol{B}] & +\eta \Delta \boldsymbol{B} \\
& +\left(\eta_{C}-\eta\right) \frac{\nabla \times([\nabla \times \boldsymbol{B} \times \boldsymbol{B}] \times \boldsymbol{B})}{B_{0}^{2}},
\end{aligned}
$$

where $\eta=\frac{c^{2}}{4 \pi \sigma}$ and $\eta_{\mathrm{C}}=\frac{c^{2}}{4 \pi \sigma_{\mathrm{C}}}$ are the Coulomb and Cowling coefficients of magnetic diffusion. It is important to note here, that besides the usual convective and magnetic diffusion terms, the induction equation (Eq. (24)) in a partially ionized plasma contains an additional term $(\propto \nabla \times([\nabla \times \boldsymbol{B} \times \boldsymbol{B}] \times \boldsymbol{B}))$. This extra term is an anisotropic magnetic diffusion resulting from the strong dissipation of the transverse to the magnetic field electric current due to ion-neutral collisions, i.e. the Cowling conductivity.

In this case, as with the traditional magnetic Reynolds number $R e_{\mathrm{m}}=\frac{4 \pi V_{0} L_{0}}{c^{2}} \sigma$, where $V_{0}$ and $L_{0}$ are a characteristic speed and spatial scale respectively, a new, "partially ionized plasma magnetic Reynolds number"

$\tilde{R e_{\mathrm{m}}}=\frac{4 \pi V_{0} L_{0}}{c^{2}} \frac{\sigma \sigma_{\mathrm{C}}}{\sigma-\sigma_{\mathrm{C}}}$

can be introduced. This new magnetic Reynolds number characterizes the relative role of the convective and the ion-neutral collisional dissipation terms in the magnetic induction equation. The ratio of the traditional $(\propto \Delta \boldsymbol{B})$ and newly appeared anisotropic magnetic diffusion terms in the Eq. (24) is

$D=\frac{c^{2} \alpha_{\mathrm{n}}}{\xi_{\mathrm{n}}^{2} B_{0}^{2} \sigma}=\frac{\sigma_{\mathrm{C}}}{\sigma-\sigma_{\mathrm{C}}}$.

In the partially ionized plasma of the solar chromosphere $(h>$ $500 \mathrm{~km}$ ), where $\sigma \gg \sigma_{\mathrm{C}}$ (see Fig. 1) and $R e_{\mathrm{m}} \sim 10^{5}-10^{7}$, the partially ionized plasma magnetic Reynolds number is $\tilde{R} e_{\mathrm{m}}=$ $\frac{4 \pi V_{0} L_{0}}{c^{2}} \sigma_{\mathrm{C}}=\frac{\sigma_{\mathrm{C}}}{\sigma} R e_{\mathrm{m}} \ll R e_{\mathrm{m}}$ and the parameter $D=\frac{\sigma_{\mathrm{C}}}{\sigma}$. This means that the traditional magnetic diffusion term can always be safely neglected, but the convective and the anisotropic magnetic diffusion terms will be of different importance in different regions of the chromosphere. In particular, in the upper chromosphere (see Fig. 1) $\tilde{R} e_{\mathrm{m}} \ll 1$ and the only third (anisotropic magnetic diffusion) term remains to be important in the induction equation. Whereas below the middle chromosphere where $\sigma_{\mathrm{C}} / \sigma$ is not very small the partially ionized plasma magnetic Reynolds number $\tilde{R e_{\mathrm{m}}}$ can be of the order or greater than unity and both terms (convective term and the anisotropic magnetic diffusion) must be included.

In the solar photosphere, where the values of $\sigma$ and $\sigma_{\mathrm{C}}$ approach each other (see Fig. 1) and $R e_{\mathrm{m}} \sim 10^{4}-10^{5}$, according to Eqs. (25) and (26), both diffusion terms in Eq. (24) can be neglected, and the induction equation will contain only the convective term in the right hand side, i.e. the induction equation will have the same form as in the case of a fully ionized plasma.

\section{Conclusion}

In this paper we have performed a comparison of the efficiency of MHD wave damping in solar plasmas due to the collisional and viscous energy dissipation mechanisms. The main conclusion which follows from this analysis is that the collisional friction damping of MHD waves is often more important than the viscous damping. This is especially true for waves propagating in the partially ionized plasmas of the solar photosphere, chromosphere and prominences.

In conclusion we would like to make just a few remarks regarding the applicability of these results and some consequences for the damping of MHD waves in the solar atmosphere.

1. Our estimations were based on the results of the MHD wave damping analysis performed within the linear approximation. This means that the expressions used above are valid only if the damping decrements $\delta=\frac{1}{2 \omega \tau}$ (or the same but with $\tilde{\delta}$ and $\tilde{\tau}$ in the case of partially ionized plasma) are much less then unity. The requirement $\delta \ll 1$ provides a limitation on the frequency range of MHD waves for which the formulas presented in this paper are formally valid. Our analysis is therefore only valid for waves with frequencies $f=\frac{\omega}{2 \pi}$ much less than a critical frequency $f_{\mathrm{c}}$. Since in the majority of cases considered in this paper the viscous damping of MHD waves is less than the collisional friction damping, i.e. $\left\{\begin{array}{c}\delta_{\text {frict }} \\ \delta_{\text {Joule }}\end{array}\right\}>\delta_{\text {visc }}$, to 

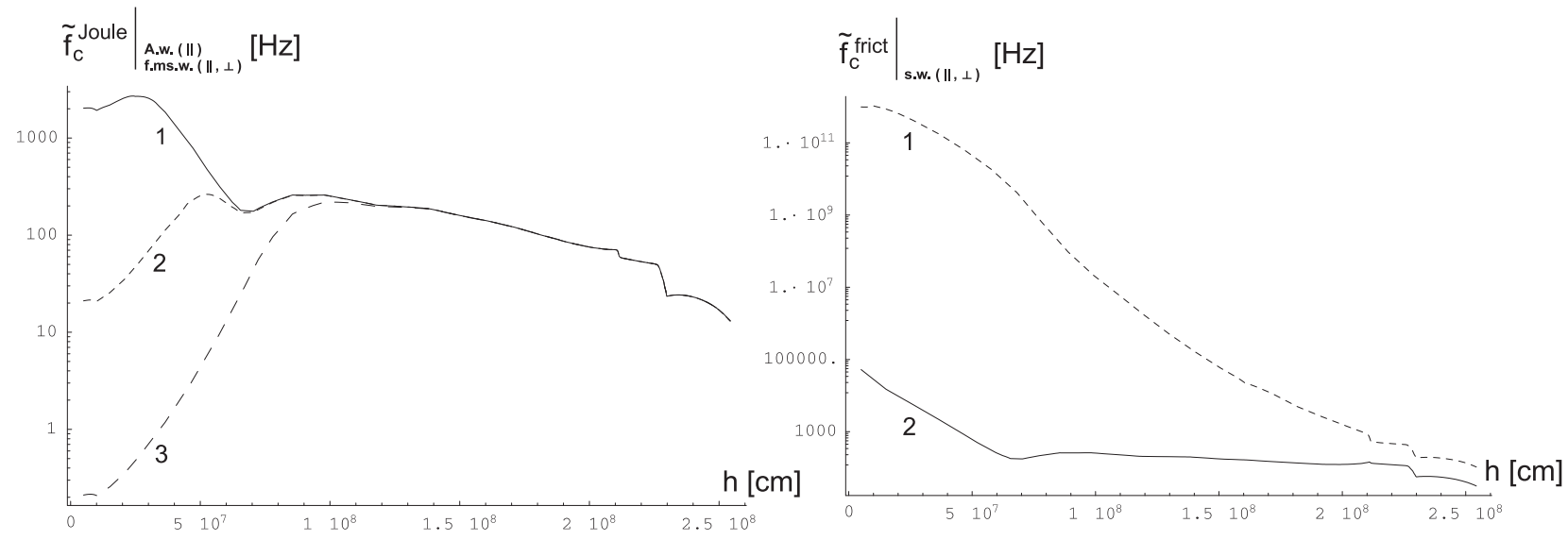

a.

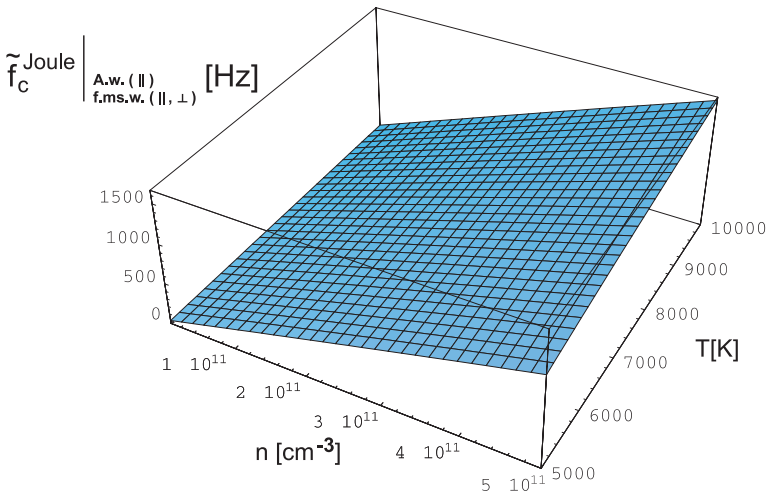

b.

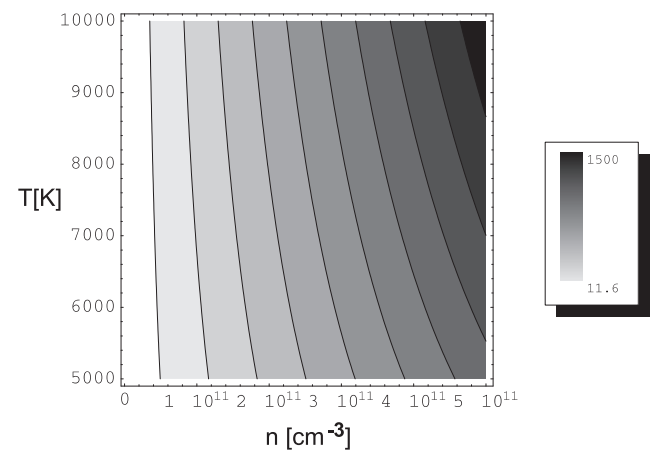

c.

Fig. 6. a) Critical frequency of the Alfvén and fast magnetoacoustic waves versus height in the low solar atmosphere for different $\left.B_{0}: 1\right) 1000 \mathrm{G}$, 2) $100 \mathrm{G}, 3) 10 \mathrm{G}$. b) Critical frequency of the acoustic wave versus height in the low solar atmosphere for 1) longitudinal (\|) and 2) transverse $(\perp)$ propagation of waves. c) The range of values of critical frequency of the Alfvén and fast magnetoacoustic waves in the prominence plasma for $B_{0}=10 \mathrm{G}$ and $\frac{n_{\mathrm{n}}}{n}=1$.

define the frequency range of validity of our analysis it makes sense to consider only the condition $\left\{\begin{array}{c}\delta_{\text {frict }} \\ \delta_{\text {Joule }}\end{array}\right\} \ll 1$, the condition $\delta_{\text {visc }} \ll 1$ will be fulfilled automatically. Equations (1), (3), (13), (15), after taking account of the appropriate dispersion relations $\omega(\boldsymbol{k})$ for particular modes, yield the following expressions for the critical frequencies:

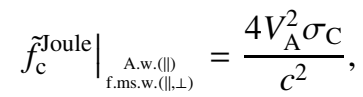

$\left.\tilde{f}_{\mathrm{c}}^{\text {frict }}\right|_{\text {s.w.(II) }}=\frac{\alpha_{\mathrm{n}} n_{0}^{2}}{\pi \rho_{0} \xi_{\mathrm{n}}^{2} n^{2}}$

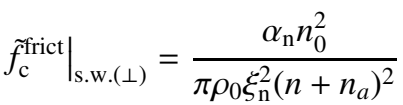

in the partially ionized plasma of the solar photosphere, chromosphere and prominences, and

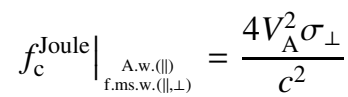

in the fully ionized coronal plasma. The dependence of

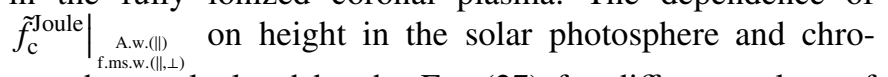
mosphere calculated by the Eq. (27) for different values of the background magnetic field $\left(B_{0}=10,100,1000 \mathrm{G}\right)$ and the atmospheric plasma parameters provided by VAL $\mathrm{C}$ model (Vernazza et al. 1981), is presented in Fig. 6a. In Fig. $6 \mathrm{~b}$ the dependencies of $\left.\tilde{f}_{\mathrm{c}}^{\text {frict }}\right|_{\text {s.w. }(\|)}$ and $\left.\tilde{f}_{\mathrm{c}}^{\text {frict }}\right|_{\text {s.w. }(\perp)}$ on height in the solar photosphere and chromosphere, defined by the Eqs. (28) and (29) are shown. The range of possible values of $\left.\tilde{f}_{\mathrm{c}}^{\text {Joule }}\right|_{\substack{\text { A.m...(\|). }(\| \perp \perp) \\ \text { in }}}$ in the prominence plasmas is presented in Fig. 6c. Here we do not give a special plot for $\left.\tilde{f}_{\mathrm{c}}^{\text {frict }}\right|_{\text {s.w.(I) }}$ in the prominence plasma, since as it follows from Eqs. (27) and (28) with $\frac{\sigma}{\sigma_{\mathrm{C}}} \gg 1$ taken into account,

$\frac{\left.\tilde{f}_{\mathrm{c}}^{\text {Joule }}\right|_{\substack{\text { f.m.w.w.(II) } \\ \text { All) }}}}{\left.\tilde{f}_{\mathrm{c}}^{\text {frict }}\right|_{\text {s.w.(II) }}}=\frac{1}{2+n_{\mathrm{n}} / n}$,

and the behaviour of $\left.\tilde{f}_{\mathrm{c}}^{\text {frict }}\right|_{\text {s.w.(II) }}$ is similar to the behaviour of

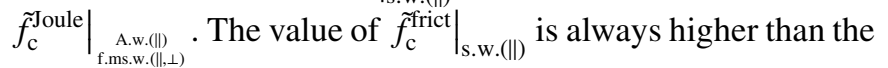


Table 2. $\left.f_{\mathrm{c}}^{\text {Joule }}\right|_{\substack{\text { f.m........(\|) }(\|) \\ \text { col }}}$ calculated for $B_{0}=10 ; 50 ; 100 \mathrm{G}$ in the corona.

\begin{tabular}{cccc}
\hline \hline & $T=10^{5} \mathrm{~K}$ & $T=5 \times 10^{5} \mathrm{~K}$ & $T=10^{6} \mathrm{~K}$ \\
\hline \multirow{3}{*}{$n=10^{8} \mathrm{~cm}^{-3}$} & $2.74 \times 10^{10}$ & $2.68 \times 10^{11}$ & $7.20 \times 10^{11}$ \\
& $2.85 \times 10^{11}$ & $6.71 \times 10^{12}$ & $1.80 \times 10^{13}$ \\
& $2.74 \times 10^{12}$ & $2.68 \times 10^{13}$ & $7.20 \times 10^{13}$ \\
\hline \multirow{3}{*}{$n=10^{10} \mathrm{~cm}^{-3}$} & $3.17 \times 10^{8}$ & $3.04 \times 10^{9}$ & $8.12 \times 10^{9}$ \\
& $7.92 \times 10^{9}$ & $7.61 \times 10^{10}$ & $2.03 \times 10^{11}$ \\
& $3.17 \times 10^{10}$ & $3.04 \times 10^{11}$ & $8.12 \times 10^{11}$ \\
\hline & $3.75 \times 10^{6}$ & $3.51 \times 10^{7}$ & $9.30 \times 10^{7}$ \\
$n=10^{12} \mathrm{~cm}^{-3}$ & $9.39 \times 10^{7}$ & $8.79 \times 10^{8}$ & $2.32 \times 10^{9}$ \\
& $3.75 \times 10^{8}$ & $3.51 \times 10^{9}$ & $9.30 \times 10^{9}$ \\
\hline
\end{tabular}

value of $\left.\tilde{f}_{\mathrm{c}}^{\text {Joule }}\right|_{\substack{\text { A.m...(\|).(\|) } \\ \text { f. }}}$. The dependence on the magnetic field in Eq. (31) is indirect via the condition $\frac{\sigma}{\sigma_{\mathrm{C}}} \gg 1$.

Some characteristic values of $\left.f_{\mathrm{c}}^{\text {Joule }}\right|_{\substack{\text { A.m.....(I) }(\|, \perp) \\ \text { c.t. }}}$ calculated for the typical coronal plasma parameters and different values of the magnetic field are presented in Table 2. For each particular density and temperature of plasma we give in the Table 2 three values of $\left.f_{\mathrm{c}}^{\text {Joule }}\right|_{\substack{\text { A.w.(I) } \\ \text { fs.w. }(\|),}}$. The upper of these values corresponds to $B_{0}=10 \mathrm{G}$, the middle, to $B_{0}=50 \mathrm{G}$, and the bottom, to $B_{0}=100 \mathrm{G}$.

2. It follows from Eqs. (13)-(15), with the corresponding dispersion relations $\omega(\boldsymbol{k})$ for the particular mode being studied, that the decrements of MHD wave collisional damping $\left\{\begin{array}{c}\tilde{\delta}_{\text {frict }} \\ \tilde{\delta}_{\text {Joule }}\end{array}\right\}$ are proportional to the wave frequency. Because of this the damping time of a wave measured in wave periods $\tilde{Q}=\tilde{\tau} f$, can be expressed via the critical frequency for the particular mode and the wave frequency:

$\tilde{Q}=(4 \pi)^{-1} \frac{\tilde{f_{\mathrm{c}}}}{f}$.

If $\tilde{Q}$ approaches unity then this will brake the validity of the above linear analysis. Using plots in Fig. 6 and Eq. (32) one can easily estimate the collisional damping time for any frequency wave.

In order for a wave with frequency $f$ to be completely damped on a particular spatial scale $L_{0}$, its propagation time $t_{\text {prop }}=L_{0} / V_{0}$ should be greater than the damping time. Using Eq. (32) this non-transparency condition can be written as

$L_{0} / V_{0}>(4 \pi)^{-1} \frac{\tilde{f_{\mathrm{c}}}}{f^{2}}$.

Here $V_{0}$ is the speed of a particular mode $\left(V_{\mathrm{A}}\right.$, or $C_{\mathrm{s}}$ in our case).

3. Under certain conditions (some cases of prominences; chromospheric plasma) more realistic modelling could require inclusion of both, collisional and viscous, mechanisms of MHD waves damping in the partially ionized solar plasmas. For a self-consistent description of MHD waves damping in this case, besides the inclusion of the appropriate terms into the momentum and energy equations, the kinetic pressure variables $p_{k}, k=\mathrm{e}, \mathrm{i}, \mathrm{n}$ in the generalized Ohm's Law (23) and Eq. (10) defining the pressure function $\boldsymbol{G}$, should be replaced by the generalized pressures $\tilde{p}_{k}=p_{k}+\tilde{\pi}_{\alpha \beta}^{k}, k=\mathrm{e}, \mathrm{i}, \mathrm{n}$, which contain the viscous stress tensors $\tilde{\pi}_{\alpha \beta}^{k}$.

However, in many cases it may be sufficient to use

$\boldsymbol{E}+\frac{[\boldsymbol{V} \times \boldsymbol{B}]}{c}=\frac{\boldsymbol{j}}{\sigma}-\frac{\xi_{\mathrm{n}}^{2}}{c^{2} \alpha_{\mathrm{n}}}[[\boldsymbol{j} \times \boldsymbol{B}] \times \boldsymbol{B}]$

and as a result Eq. (24) for the induction equation (Khodachenko \& Zaitsev 2002). The conditions which must be met for such a simplification were detailed in the previous section. Note further that if the only currents in the system are perpendicular to the magnetic field, as is the case for a pure Alfvén wave in simple geometry, then it is valid to simplify this further and use $\mathbf{E}+\frac{[\boldsymbol{V} \times \boldsymbol{B}]}{c}=\frac{\boldsymbol{j}}{\sigma_{\mathrm{C}}}$ as the generalized Ohm's Law. However, care must be taken to avoid this final simplification if there are parallel currents in the system.

In the corona it is common to treat the conductivity as a scalar since the perpendicular and parallel components only differ by a factor of about 2 . This is especially true in numerical simulations where the numerical resistivity exceeds the real resistivity by many orders of magnitude. The situation in the chromosphere is quite different with the perpendicular resistivity $\left(1 / \sigma_{\mathrm{C}}\right)$ exceeding the parallel component $(1 / \sigma)$ by many orders of magnitude. The Cowling conductivity is so small that it may be possible to use the real value in numerical simulations. To treat the conductivity as a scalar in such circumstances would damp any parallel currents and may lead to an erroneous magnetic field structure.

4. In this paper we did not consider one more important mechanism of MHD waves energy dissipation - thermoconductivity. Preliminary rough estimations indicate that sometimes (in particular in the low solar atmosphere) the thermoconductivity effects could be of the same importance for MHD waves damping as the collisional friction, and appear to be more important than viscosity effects.

Acknowledgements. This work was supported by the Academic Research Collaboration Programme of the British Council in Austria and the ÖAD-RFBR Scientific and Technical Collaboration Program (No.I.21/04). M. L. Khodachenko is grateful to the Austrian Academy of Sciences (ÖLZELT grant) and grant FWF (P16919-N08) for supporting his work. The authors express their special thanks to V. V. Zaitsev for useful comments and discussions.

\section{References}

Antiochos, S. K., MacNeice, P. J., Spicer, D. S., \& Klimchuk, J. A. 1999, ApJ, 512, 985

Aschwanden, M. J., Newmark, J. S., Delaboudiniere, J.-P., et al. 1999a, ApJ, 515, 842

Aschwanden, M. J., Fletcher, L., Schrijver, C. J., \& Alexander, D. 1999b, ApJ, 520, 880

Aschwanden, M. J., Nightingale, R. W., \& Alexander, D. 2000, ApJ, 541,1059

Aschwanden, M. J., Schrijver, C. J., \& Alexander, D. 2001, ApJ, 550, 1036 
Bakhareva, N. M., Khodachenko, M. L., \& Zaitsev, V. V. 1992, Sol. Phys., 139, 299

Braginskii, S. I. 1965, Transport processes in a plasma, in Reviews of plasma physics (New York: Consultants Bureau), 1

Cowling, T. G., Magnetohydrodynamics (New York: Interscience), 1957

DePontieu, B., \& Haerendel, G. 1998, A\&A, 338, 729

DePontieu, B., Martens, P. C. H., \& Hudson, H. S. 2001, ApJ, 558, 859

Frank-Kamenetskii, D. A. 1961, Sov. Phys. Techn. Phys., 5, 842

Goodman, M. L. 2000, ApJ, 533, 501

Goodman, M. L. 2001, Space Sci. Rev., 95, 79

Gordon, B. E., \& Hollweg, J. V. 1983, ApJ, 226, 373

Haerendel, G. 1992, Nature, 360, 241

Hollweg, J. V. 1986, JGR, 91, 4111

Hollweg, J. V., in Mechanisms of Chromospheric and Coronal Heating, ed. P. Ulmschneider, E. R. Priest, \& R. Rosner (Berlin: Springer), 423, 1991

James, S. P., \& Erdélyi, R. 2002, A\&A, 393, L11

James, S. P., Erdélyi, R., \& De Pontieu, B. 2003, A\&A, 406, 715

Karpen, J. T., Antiochos, S. K., Hohensee, M., Klimchuk, J. A., \& MacNeice, P. J. 2001, ApJ, 553, L85
Khodachenko, M. L. 1996, Astron. Rep., 40, 273

Khodachenko, M. L., \& Zaitsev, V. V. 1992, Sov. Astron., 36, 81

Khodachenko, M. L., \& Zaitsev, V. V. 2002, Ap\&SS, 279, 389

Landau, L. D., \& Lifshits, E. M. 1987, Fluid Mechanics (Oxford: Pergamon Press)

Nakariakov, V. M., Ofman, L, DeLuca, E., Roberts, B., \& Davila, J. M. 1999, Science, 285, 862

Nakariakov, V. M., Verwichte, E., Berghmans, D., \& Robbrecht, E. 2000, A\&A, 362, 1151

Narain, U., \& Ulmschneider, P., 1996, Space Sci. Rev., 75, 453

Ofman, L. 2002, ApJ, 568, L135.

Osterbrock, D. 1961, ApJ, 143, 347

Piddington, J. H. 1954, MNRAS, 114, 638

Piddington, J. H. 1956, MNRAS, 116, 314

Priest, E. R., Foley, C. R., Heyvaerts, J., et al. 1998, Nature, 393, 545

Spadaro, D., Lanza, A. F., Lanzafame, A. C., et al. 2003, ApJ, 582, 486

Spitzer, L. 1962, Physics of fully ionized gases (New York: Interscience)

Tsiklauri, D., \& Nakariakov, V. M. 2001, A\&A, 379, 1106

Vernazza, J. E., Avrett, E. H., \& Loeser, R. 1981, ApJS, 45, 635

Zaitsev, V. V., \& Stepanov, A. V. 1992, Sol. Phys., 139, 343 